\title{
Article
}

\section{A System for Monitoring Acoustics to Supplement an Animal Welfare Plan for Bottlenose Dolphins}

\author{
Brittany L. Jones ${ }^{1, *(\mathbb{D}}$, Michael Oswald ${ }^{2}$, Samantha Tufano ${ }^{1}$, Mark Baird ${ }^{1}$, Jason Mulsow ${ }^{1}$ \\ and Sam H. Ridgway ${ }^{1}$ (D) \\ 1 National Marine Mammal Foundation, San Diego, CA 92106, USA; sam.tufano@gmail.com (S.T.); \\ mark.baird@nmmf.org (M.B.); jason.mulsow@nmmf.org (J.M.); sam.ridgway@nmmf.org (S.H.R.) \\ 2 Scottish Oceans Institute, St Andrews University, St Andrews KY168LB, UK; m2oswald@gmail.com \\ * Correspondence: brittany.jones@nmmf.org
}

Citation: Jones, B.L.; Oswald, M.; Tufano, S.; Baird, M.; Mulsow, J.; Ridgway, S.H. A System for Monitoring Acoustics to Supplement an Animal Welfare Plan for Bottlenose Dolphins. J. Zool. Bot. Gard. 2021, 2, 222-233. https:// doi.org/10.3390/jzbg2020015

Academic Editor: Lance Miller

Received: 18 March 2021

Accepted: 27 April 2021

Published: 29 April 2021

Publisher's Note: MDPI stays neutral with regard to jurisdictional claims in published maps and institutional affiliations.

Copyright: (c) 2021 by the authors. Licensee MDPI, Basel, Switzerland. This article is an open access article distributed under the terms and conditions of the Creative Commons Attribution (CC BY) license (https:/ / creativecommons.org/licenses/by/ $4.0 /)$.

\begin{abstract}
Animal sounds are commonly used by humans to infer information about their motivations and their health, yet, acoustic data is an underutilized welfare biomarker especially for aquatic animals. Here, we describe an acoustic monitoring system that is being implemented at the U.S. Navy Marine Mammal Program where dolphins live in groups in ocean enclosures in San Diego Bay. A four-element bottom mounted hydrophone array is used to continuously record, detect and localize acoustic detections from this focal group. Software provides users an automated comparison of the current acoustic behavior to group historical data which can be used to identify periods of normal, healthy thriving dolphins, and allows rare instances of deviations from typical behavior to stand out. Variations in a group or individual's call rates can be correlated with independent veterinary examinations and behavioral observations in order to better assess dolphin health and welfare. Additionally, the monitoring system identifies time periods in which a sound source from San Diego Bay is of high-enough amplitude that the received level at our array is considered a potential concern for the focal animals. These time stamps can be used to identify and potentially mitigate exposures to acoustic sources that may otherwise not be obvious to human listeners. We hope this application inspires zoos and aquaria to innovate and create ways to incorporate acoustic information into their own animal welfare management programs.
\end{abstract}

Keywords: animal welfare; acoustic monitoring; vocal behavior; vocal biomarkers; marine mammal; bioacoustics; communication; distress call

\section{Introduction}

Methods for collecting, analyzing and classifying underwater sounds have been rapidly improving over the last 50 years. The development of compact, mobile, and often autonomous recording systems has given us unparalleled access to information on soundscapes, anthropogenic noise, and animal presence and density. Although they are quickly becoming a major aspect of marine conservation efforts, the potential for using these methods to enhance animal welfare in managed care facilities has not yet been widely implemented [1].

McLoughlin et al. [1] review the current status of animal welfare monitoring and insist that vocal behavior is an underutilized welfare indicator amongst both terrestrial and aquatic species. Brando et al. [2] report that the best practice study of cetacean welfare biomarkers to date is Clegg et al. [3]. While Clegg et al. provide an index of 36 welfare indicators, none of the measures consider the acoustic behavior of an individual, group or population. Social structure is often considered an important measure of welfare, however, acoustic access to one another may be as important as physical access in this context.

Bottlenose dolphins are arguably the most well studied marine mammal species in terms of acoustic behavior [4]. This species has a rich and varied vocal behavior. For example, two female Tursiops truncatus were recorded for $24 \mathrm{~h}$ every two weeks for a year [5] and 
their whistle emissions ranged from 1682 to 6793 whistles for 24 hour periods. Ridgway [6] furthered that whistle rate changes dependent on health and reproductive cycle of the females. To date, a few vocalization types have been validated to indicate the presence of important welfare related behaviors such as the occurrence of burst pulses during aggressive interactions [7], and the persistent use of signature whistles when an animal is in distress (i.e., a distinctive whistle contour that is used by an individual to broadcast its identity) [8-10].

Ridgway [7] found that dolphins produce pulsed 'calls' during periods of aggression and agitation. Overstrom [7] similarly observed that burst pulse vocalizations are typically emitted in aggressive chases, open mouthing behaviors, arched posturing, jaw clapping, and head to head encounters. Blomqvist and Amundin [11], also found that as aggressive interactions between two dolphins reached a climax, such as a physical fight, so did the production of high intensity burst pulse vocalizations.

Distress calls are emitted by many animal species in dire contexts such as danger, severe injury or illness and function to warn and/or elicit help from conspecifics [12]. Lilly [10] was the first to report that dolphins utilize distress calls, which are repeated whistles used to elicit attention and aid from other animals. Caldwell and Caldwell [8] furthered that the whistle type emitted by a dolphin in distress is its signature whistle [8] and that the signature whistle is loud and incessant during these contexts. Kuczaj et al. [9] recorded a dying dolphin producing one stereotyped whistle contour relentlessly in its final hour. Having a validated acoustic indicator for events such as agnostic social interactions and/or an instance of distress in bottlenose dolphins provides a unique opportunity for acoustic monitoring to be used as a tool for the early identification and possible prevention of rare and unexpected instances of an illness or injury.

Here, we present a new tool, National Marine Mammal Foundation's Welfare Acoustic Monitoring System (NMMF WAMS), hereafter referred to as WAMS, for open-source use in PAMGuard [13]. PAMGuard is an open-source program used to collect and analyze marine mammal acoustic data, and is designed to provide a flexible, module-based and user-friendly platform to assist in passive acoustic monitoring of marine mammals. The software's modular framework allows users to add components depending on needs and specifications of different project goals, including detectors and classifiers which can be used to study various cetacean species. This structure allows 'plug-in' modules to easily be developed and integrated by the wider research community. PAMGuard is a widely used platform for marine mammal mitigation and monitoring in the offshore seismic survey and construction industries, but its use in monitoring discrete animal groups has been minimal to date.

This manuscript highlights the integrative quality of WAMS. WAMS synthesizes previously developed plug-ins with new features and user-customized interfaces for creating a tool for monitoring acoustic data from a group of dolphins in managed care. We describe the current deployment of WAMS for monitoring the acoustic behavior of 10 bottlenose dolphins at the Navy Marine Mammal Program (MMP) in San Diego, CA, U.S. The WAMS plug-in within open source PAMGuard receives acoustic data from a four-channel modular, bottom mounted array in San Diego Bay. The primary goal of this array deployment and program development is to allow researchers, veterinarians and training staff to use the acoustic behavior of the dolphins as indicators of motivational state, arousal level, and health status. The research described above has suggested that if an animal were to be in distress (an extremely rare occurrence in this group), it might vocalize at a high rate and intensity. The round the clock monitoring of typical and normal behavior establishes a rich baseline for a rare abnormal event to stand out against. Early detection of any abnormal event could prove to be a valuable long-term tool to support that goal. While specific data on dolphin welfare are outside of the scope of this system description, we deliver a theoretical basis for listening tools to inform and improve animal welfare programs. 


\section{System Description: Methods and Results}

The WAMS plugin provides a method of logging audio detections of interest and providing both an instantaneous count in the current time period, as well as a historical mean count to compare against. The size of the time period is user-adjustable. The present study uses an hourly time bin for the display, and also uses five minute time bins for more finescale analyses. WAMS can run in both real time and during post-processing of previously recorded audio data, and can generate reports using operator-customized templates.

WAMS is currently acoustically monitoring a group of dolphins' whistling behavior $24 \mathrm{~h}$ a day, seven days a week. This tool is currently being used daily across a multitude of contexts to incorporate this often overlooked behavior into the dolphins' overall animal care and welfare program. This program takes an ecologically balanced approach to consider information on individual dolphin behavior, dolphin interactions, and the interaction of dolphins with environmental sounds.

Some examples of its incorporation in the MMP to date:

1. Monitors for the presence of 'normal, average' communicative behavior for a thriving group of dolphins;

2. Allows for the identification of seasonal, diurnal, and hormonal trends in vocal behavior following integration with veterinary data;

3. Gives insight into the dolphins' habituation to novel situations, including novel environments during Navy dolphin deployments;

4. Alerts animal care professionals to times of abnormally high and/or low rates of vocalizations;

5. Allows caretakers to localize dolphins when doing open water training exercises outside of enclosures;

6. Provides insight to the valence and arousal levels of dolphins during in-session training settings and off-session social interactions;

7. Allows for identification of noise conditions that with potential for masking dolphin vocalizations and/or acoustic behavioral response to anthropogenic noise in San Diego Bay.

Below we outline the specifications of the hardware and software that are developed and implementing in achieving the above goals.

\subsection{Hardware}

Recordings are made using a four element, bottom mounted stationary hydrophone array. Four HTI-99-HF hydrophones (High-Tech Inc, Long Beach, MS, U.S., 2 Hz-125 kHz frequency response with integrated preamplifier) are mounted in a tetrahedral shape, with 3 hydrophones making up the base and the 4th hydrophone raised in the center. Spacing between any pair of hydrophones is $150 \mathrm{~mm}$. This shape eliminates the right/left ambiguity common to linear and planar arrays. The frame holding the hydrophones is made from titanium and mounted in a larger frame made of PVC pipe. The frame is filled with water and anchored to the ocean floor with diver weights. Each hydrophone cable is 150 meters long and terminates at a 4 channel SAIL DAQ card (SA Instrumentation) in a custom-built electronics enclosure with filtered power and amplified line-out jack. Signals are passed from the enclosure to a DELL laptop via USB cable. The SAIL DAQ unit and laptop are stored in a pelican case inside an above water hut located on a floating dock at NIWC Pacific in San Diego, California (Figure 1).

\subsection{Study Group}

This array is deployed at the U.S. Navy MMP in San Diego, CA, U.S. The focal dolphins in the present study are housed in natural seawater enclosures in San Diego Bay. Males and females are located in separate enclosures approximately $10 \mathrm{~m}$ from one another. In order to test the efficacy of this monitoring system, it is programmed to record one of the two enclosures housing the total dolphin group ( $N=10$ dolphins) (Figure 2). This was implemented to ensure successfully localization and development of the program before 
extending the monitoring system to the larger group. In order to do this, an angle veto is being used. Angles of arrival from the calling animal to the array are calculated for all signals. However, the program only 'counts' whistles with angles of arrival consistent with the direction of the focal animals (Figure 2). These angles were established by playback experiments using whistle recordings emitted throughout the enclosure and determining the angles of arrival that correspond to the focal dolphins. The speaker was placed $1 \mathrm{~m}$ from the array in 10 degree increments 360 degrees around the system. This determined the directionality of the array, confirmed successful localization capabilities in the present environment, and provided the angles of arrival that are used as input into the angle veto of the WAMS module.

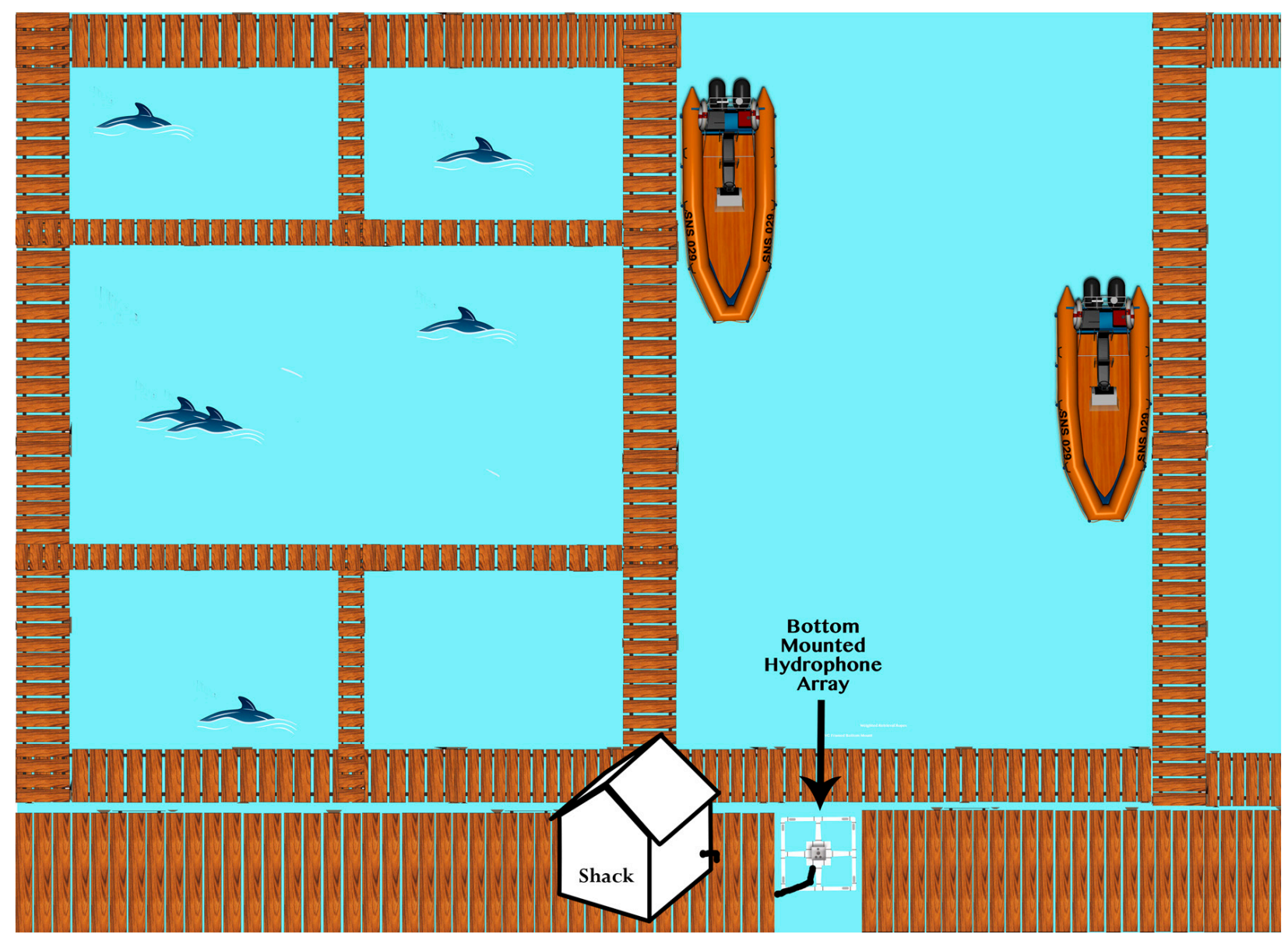

Figure 1. Aerial schematic of the general location of the bottom mounted hydrophone array in relation to the focal group of dolphins, and the shack that houses the sound card and computer components.

\subsection{Software}

The WAMS plugin has been designed to work in conjunction with existing core PAMGuard modules. PAMGuard is a modular system that receives audio input and then processes that input in a variety of ways depending on the needs of the operator. All aspects of data conversion, manipulation, analyses, and visual depiction must be explicitly defined by the user. A typical PAMGuard configuration may consist of the following modules: Array Manager, Sound Acquisition, Sound Recorder, Fast-Fourier transform (FFT) Engine, Whistle \& Moan Detector, and WAMS plug-in. In addition, a Database module and Binary Storage module must be included in order to store output data from the detector and WAMS modules. A User Display module is also typically included to display a spectrogram of the audio signal. 


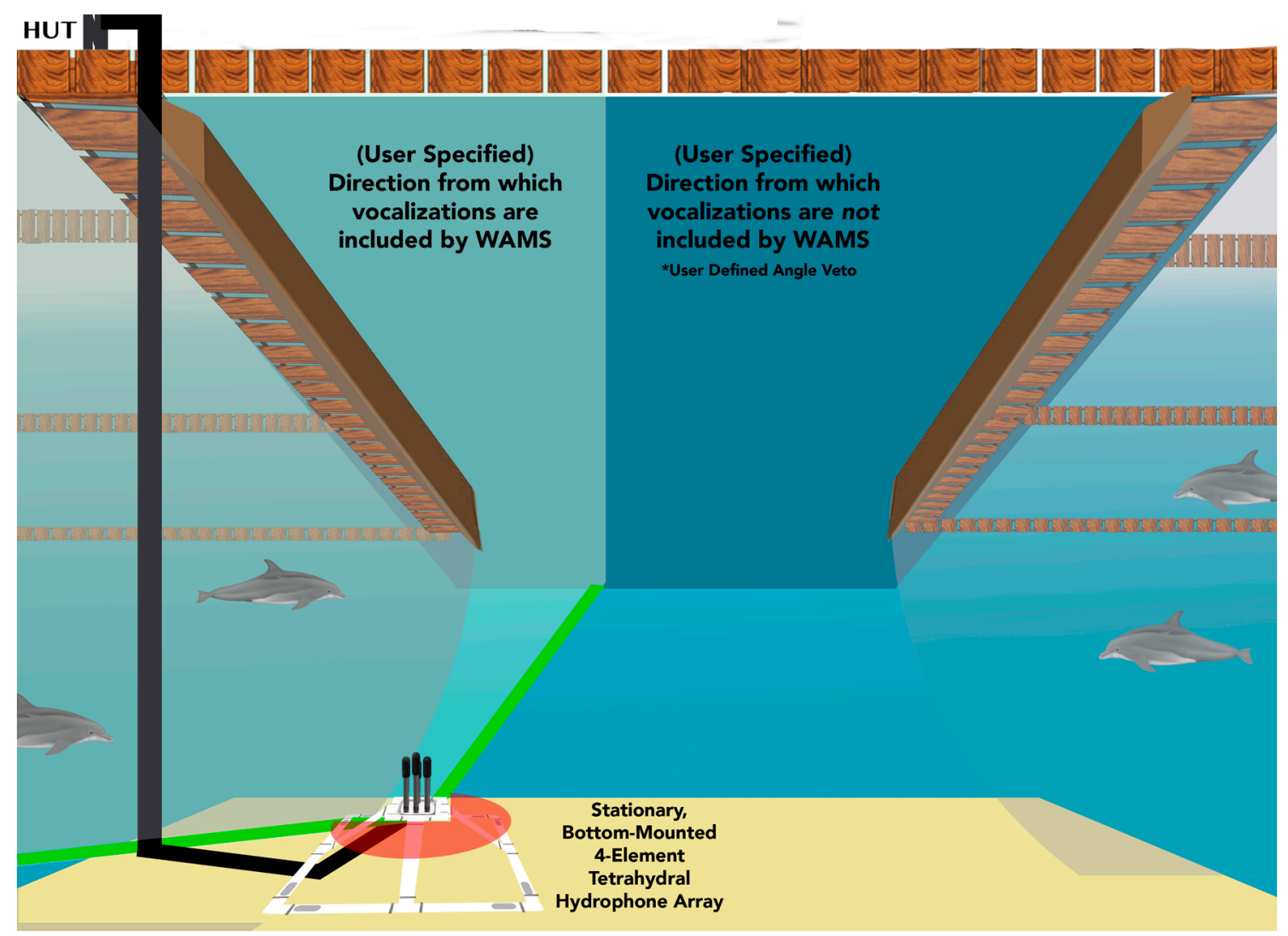

Figure 2. Underwater schematic of the bottom-mounted hydrophone array deployed at the MMP. The two natural seawater enclosures are separated in the center by a $10 \mathrm{~m}$ wide channel which separates two groups of dolphins. The angle veto in WAMS excludes whistle detections that originate outside of the delineated range.

The Array Manager (Figure 3) must be configured with details of the hydrophone array used to collect the audio data. Arrays may be stationary (such as a fixed mooring) or transient (such as a towed array), and positional details of each hydrophone are subsequently used when calculating bearings of detected sounds. The WAMS plugin will work with a single hydrophone, however in order to calculate bearing to the sound source at least 2 hydrophones are required.

The Sound Acquisition module configuration determines the input sound source. This can be a real-time audio from a sound card or network source, or a pre-recorded audio file. The Sound Recorder module will save the audio stream to a hard drive as a .wav file. The user is able to limit the size of the .wav file by either specifying a maximum size in bytes or a maximum duration. The FFT Engine (Figure 4) converts the time-domain data into frequency-domain data suitable for spectrogram display.

The previously developed PAMGuard 'Whistle and Moan Detector' module (hereafter referred to as 'the whistle detector') is used to detect the dolphin whistles. The whistle detector module uses a six-stage process to detect whistles in the frequency-domain data that is output from the FFT Engine. A description of the whistle detector's algorithms can be found in Gillespie et al. [13]. Rankin et al. [14] provides the settings used for detection of whistles during passive acoustic monitoring efforts off the coast of California. We used these parameters as a starting place and further validated that the parameters were successful in identifying whistle behavior in this group. The only change that we made was to concentrate on the frequency range $3 \mathrm{kHz}-20 \mathrm{kHz}$ (Table 1) which reduced false detections from anthropogenic noise especially in the lower frequencies. While dolphin whistles can reach frequencies outside of this range, for our population, it would be very rare for an entire whistle to do so. 


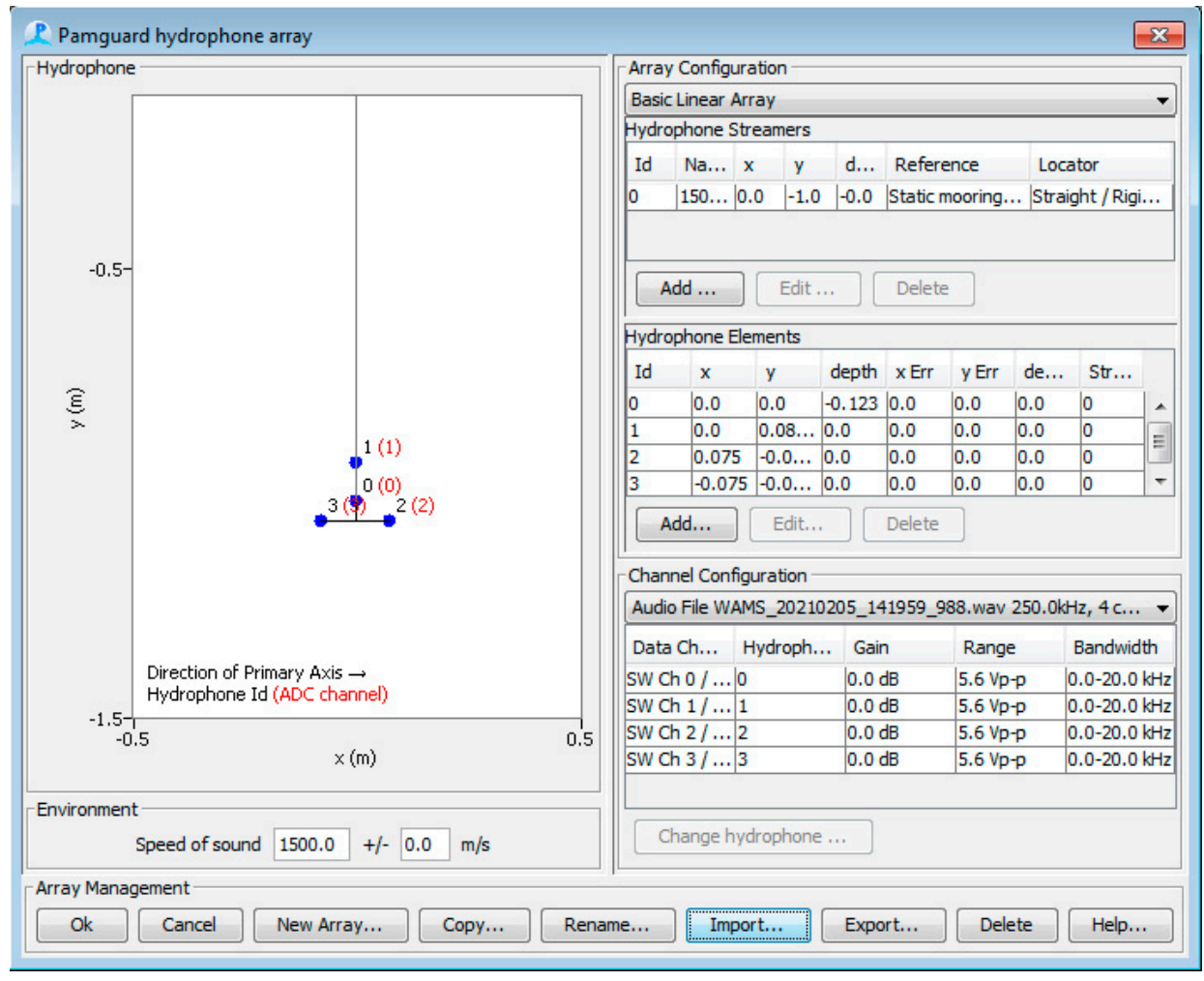

Figure 3. An example of the array manager configuration for the 4-element tetra-hedral bottom mounted hydrophone array used in the current MMP deployment.

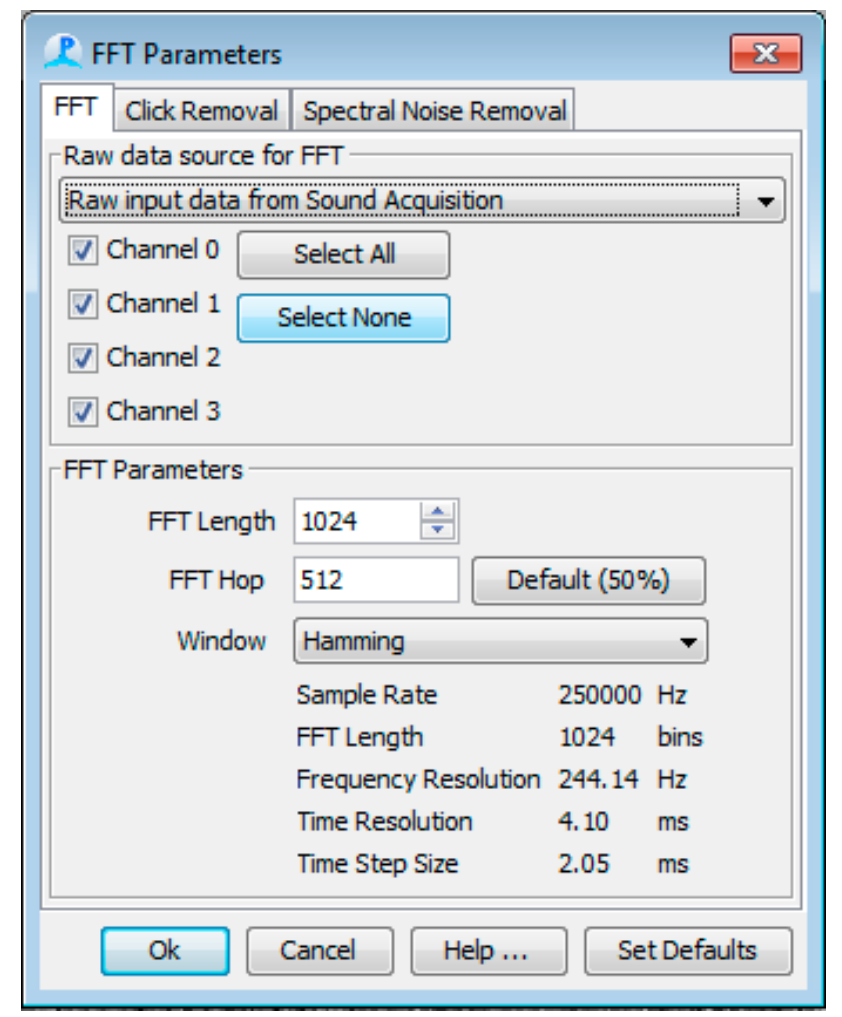

Figure 4. An example of the FFT Parameters module in PAMGuard. This module allows the user to take the raw input from the sound acquisition module and takes an FFT dependent on the user inputs for length, FFT hop size, overlap, and window type. 
Table 1. The whistle detector settings that were used in the present study to detect bottlenose dolphin whistles. Adapted from Rankin et al. [15]. For definitions of the column headings please see Appendix A. For additional details on what each setting does see Gillespie et al. [13].

\begin{tabular}{cccccccc}
\hline $\begin{array}{l}\text { Maximum } \\
\text { Frequency }\end{array}$ & $\begin{array}{c}\text { Minimum } \\
\text { Frequency }\end{array}$ & $\begin{array}{c}\text { Connection } \\
\text { Type }\end{array}$ & $\begin{array}{c}\text { Minimum } \\
\text { Length }\end{array}$ & $\begin{array}{c}\text { Minimum } \\
\text { Total Size }\end{array}$ & Crossing/Joining & $\begin{array}{c}\text { Maximum } \\
\text { Cross } \\
\text { Length }\end{array}$ & $\begin{array}{c}\text { Median } \\
\text { Filter } \\
\text { Length }\end{array}$ \\
\hline $20 \mathrm{kHz}$ & $3 \mathrm{kHz}$ & 8 sides & 10 slices & 50 & Relink & 5 slices & $\begin{array}{c}\text { Subtraction } \\
\text { Constant }\end{array}$ \\
\hline
\end{tabular}

When a whistle is detected, the WAMS module is notified and increases the count in the current time period by 1 . When a time period has elapsed, the mean value of the typical whistle rate during that time period is recalculated based on the most recent count combined with all historical data for that time period. Therefore, the mean of each designated time period is continually updating. The count and ancillary information are logged both to the database and a .csv file. The information can also be displayed in a User Tab in both tabular and graphical formats (see Figure 5 for an example of the real time display).

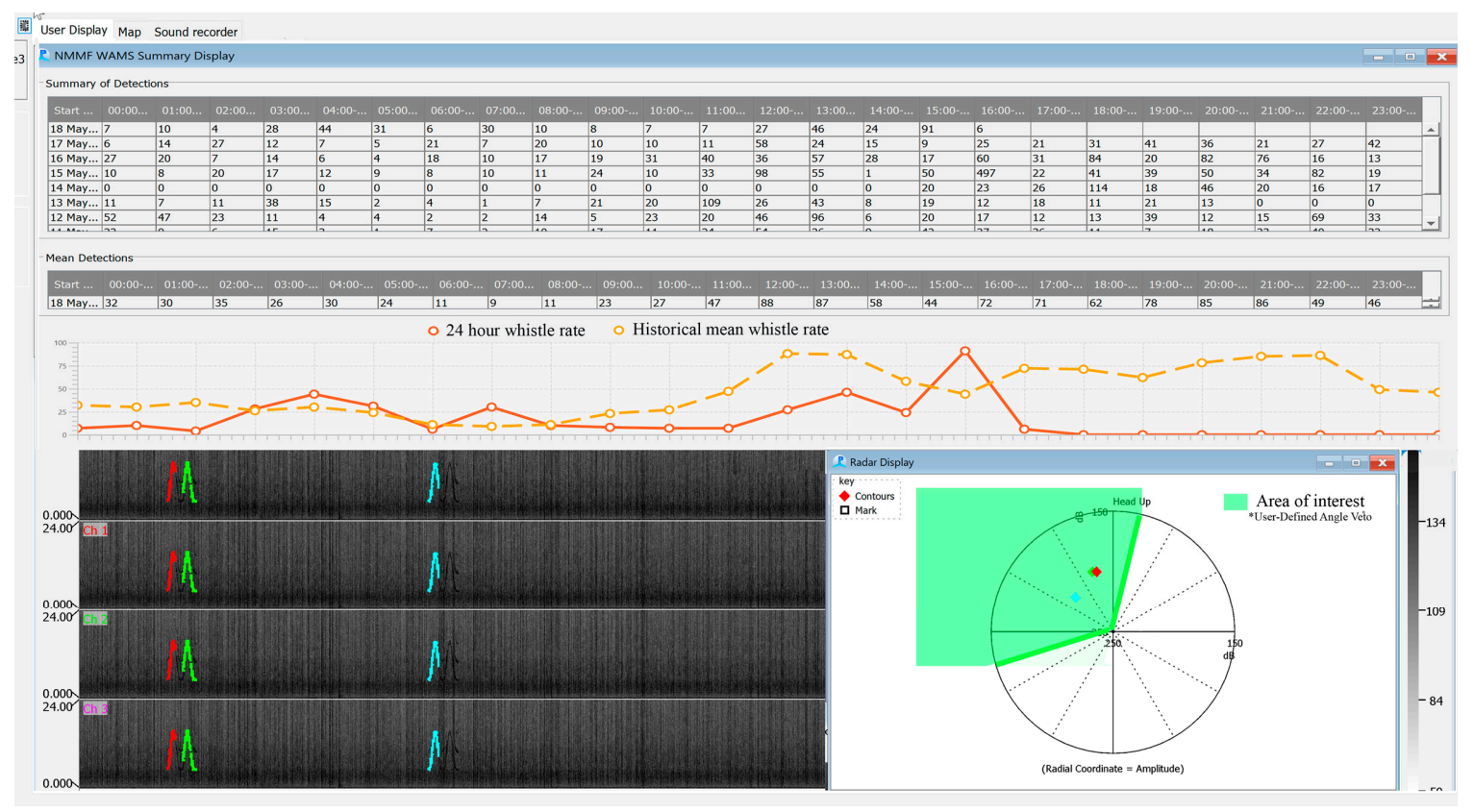

Figure 5. Interface of the WAMS module running in PAMGuard. The WAMS Summary Display on the top panel recordings the whistle detections in each hour of every day. The historical means are plotted below in orange (dashed) and the current 24-h detections are plotted in red (solid). In the lower left are the spectrograms of the four channels recording from the hydrophone array. The whistle detector detects and traces whistles (colored lines) and the corresponding colored diamonds on the radar display show the localization of the detection. An angle veto is used to only include detections from the direction highlighted in green in the radar display during daily operations.

The PAMGuard Alarm module (Figure 6) is a helpful addition to the configuration. In this study, the Alarm module receives information from the WAMS module. When the counts exceed a certain threshold value over a specified time period (e.g., 100 whistles in $5 \mathrm{~min}$ ), the Alarm module can respond by sending an output signal through the serial port, displaying a message on the screen, sounding an audible alarm, and/or sending an email alert to a specified email address. The alarm module was updated for optimal use with WAMS. When an alarm is triggered due to a high detection rate, an email with a summary of the event sent to the designated address, with a screenshot of the PAMGuard display window during that time period attached. This gives almost instantaneous feedback to an interested party that an alarm has been triggered but also allows the operator to immediately see the cause of the alarm and the bearing from which the detections are 
coming from. In our case, we are able to see what location in the lagoon and what whistle type is being emitted at an abnormally high rate and can respond appropriately.

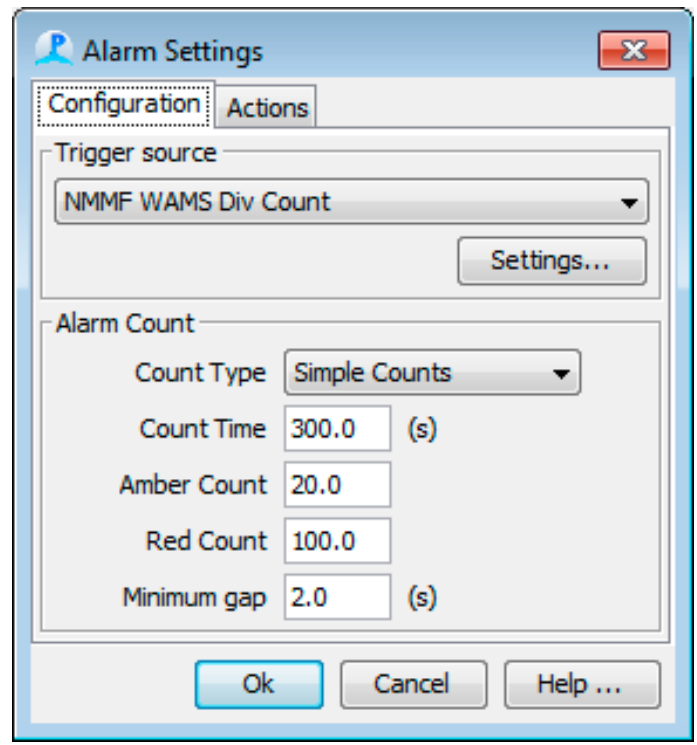

Figure 6. An example of the alarm module in PAMGuard. This module allows the user to identify whistle rates of interest and have the program trigger an email alert to be sent to the researcher when the detections surpass a user defined threshold. When the threshold is surpassed the program records an image of the current spectrogram and attaches it to the email for review by the recipient.

The WAMS plugin provides the user with a number of options to better serve the specific application. The WAMS plugin is able to accept notifications from any of the detector modules (e.g., Click Detector, Whistle and Moan Detector) within PAMGuard. Many detector modules calculate bearing angles when there are two or more hydrophone elements in the array. The bearing for each detection is reported in the database regardless of whether it was excluded from the WAMS counts to ensure that no data are lost.

In the presence of signals with harmonic components the whistle detector will often treat the individual harmonics as separate whistle contours, in turn, artificially increasing the WAMS count (example in Figure 7). In order to minimize this, a simple Harmonic Detector can be configured within the WAMS plugin to disregard contours with similar starting/ending points and require a minimum amount of time between contours to be considered separate detections. Upon turning on the harmonics detector, the user is asked to input a threshold (ms) for the minimum time difference between two start times and/or between two end times. There is also an option to set a minimum time gap between two detections (ms). As a new contour is detected, the starting time is compared to the starting times of the whistle contours currently held in memory. If the difference in time is less than the threshold value specified by the user, that whistle is discarded as a harmonic. The process is then repeated with the end time. Turning on the harmonic detector ensures that when two traces are closely related in time, they are not counted as more than one whistle.

WAMS can generate reports in a Microsoft Word .docx format. The report will contain the counts and alarms during the specified time period and provide users with summary data at user-defined intervals.

Information supplemental to vocal rates can also be gained through analysis of continuous acoustic data from the array hydrophones. In the current implementation of the WAMS system, ambient acoustic data are immediately analyzed using custom LabVIEWbased software (National Instruments Corporation, Austin, TX, U.S.) after being stored to hard disk (Figure 8). This analysis includes simple threshold detectors for spectral levels within frequency regions of interest, as well as overall "weighted" levels based on the 
hearing capabilities of dolphins (i.e., the "high-frequency cetacean" weighting function in Southall et al. [16]). Both of these methods can be used to present a summary of time periods in which acoustic events of interest occur (while filtering out normal ambient conditions that fall below thresholds). This is especially useful considering that events within the typical audible range of dolphins may not be audible to humans, and an assessment of an overall "weighted" level based on dolphin hearing may not readily available from either listening or visual review of a spectrogram [15]. Time stamps of the periods in which thresholds are exceeded can then be used to identify and mitigate sources of concern, and to cross reference with vocal behavioral patterns.

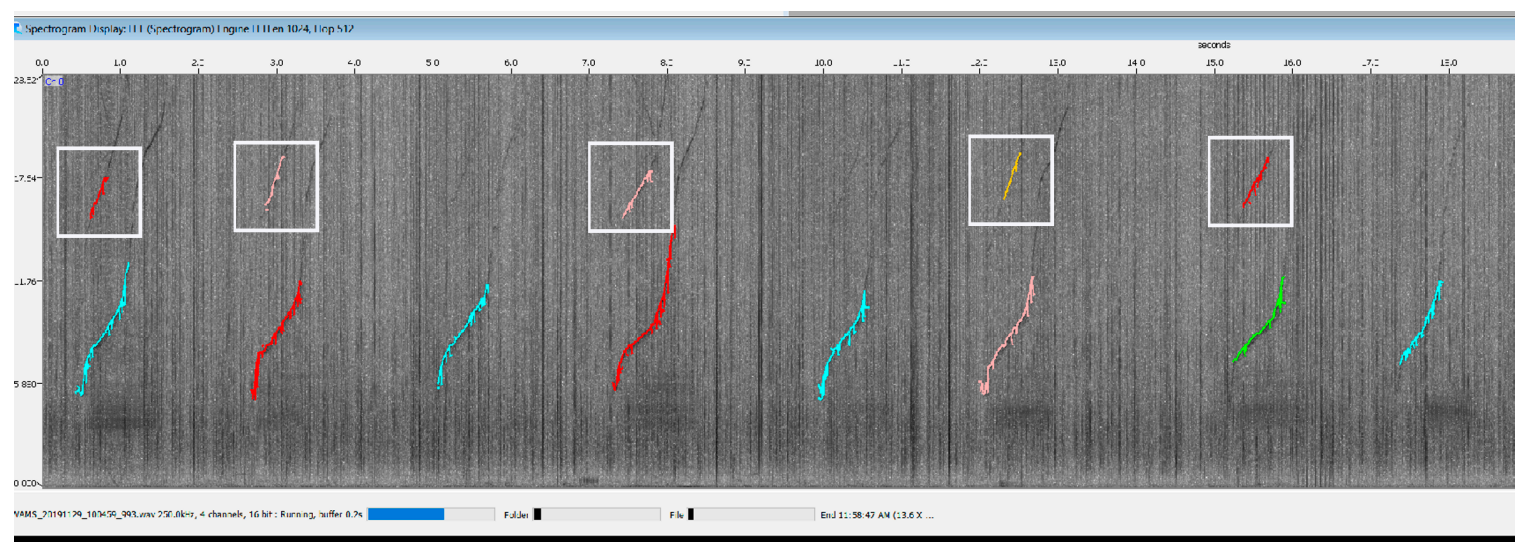

Figure 7. A spectrogram of whistle detections in WAMS using the whistle detector (time (s) on the $\mathrm{x}$-axis, frequency (kHz) on the y-axis). By toggling on the harmonic detector, the traces that are within the white boxes will still be traced, but not included in the whistle counts as they are identified as harmonics of a different whistle detection. With the harmonic detector turned off these would be counted as whistle detections in addition to the fundamental contours traced below them. WAMS would count the current segment as 8 whistles with the harmonic detector turned on and 13 with it turned off.

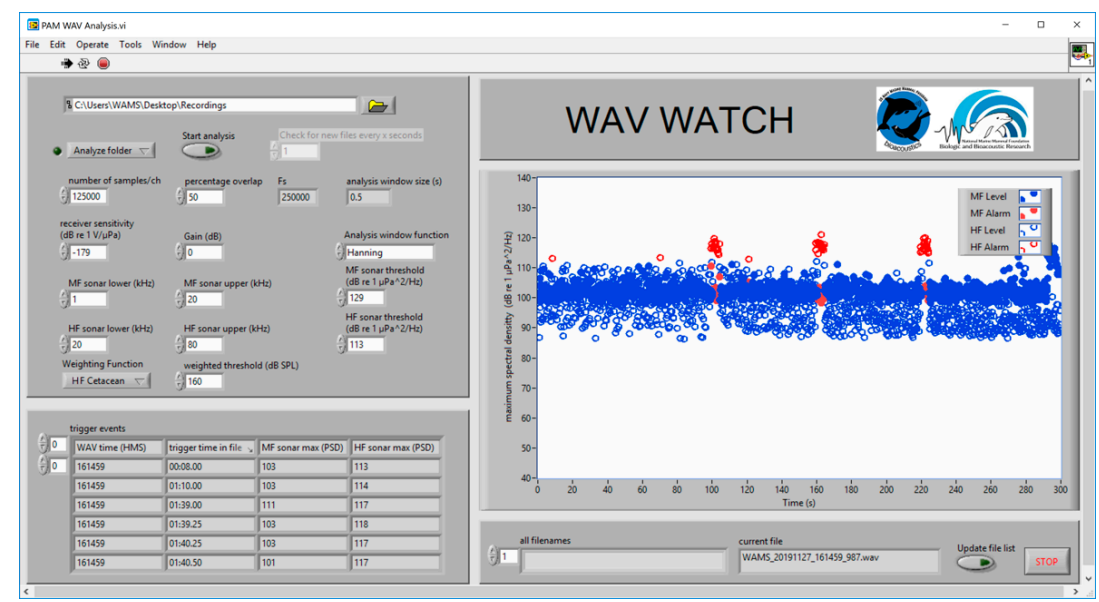

Figure 8. The LabVIEW-based module that evaluates the previous five minutes of data recorded using WAMS for spectral levels that exceed a user defined threshold and are weighted by the high-frequency cetacean weighting curve function [16]. The blue dots indicate a level less than the user-defined threshold (i.e., Mid-Frequency (1-20 kHz) set at $129 \mathrm{~dB}$ re $1 \mu \mathrm{Pa}^{2 /} \mathrm{Hz}$; High-Frequency (20-80 kHz) set at $113 \mathrm{~dB}$ re $1 \mu \mathrm{Pa}^{2 /} \mathrm{Hz}$ ), and a red dot would indicate a sound source that is a level louder than the threshold. If the dot is an open circle (red or blue) it is measuring the signal in the high frequency range, if the dots are closed (red or blue) it is measuring the signal in the mid-frequency range. 


\section{Discussion}

Here, we present a welfare acoustic monitoring system for using acoustic data to help inform researchers, veterinary staff and animal caretakers about the well-being of dolphins in managed care. By providing both five minute and hourly counts of acoustic detections and automatically comparing those counts to historical data it can alert users to rare instances of high vocal rates relative to their typical output.

The ability to manipulate settings for each array configuration, soundscape and project goal allows for flexibility in application. Testing of WAMS for a multitude of other welfare monitoring goals such as the occurrence of aggression, early identification of ailment, and the habituation to novel experiences in bottlenose dolphins has already begun.

Equally as important is the identification of positive-welfare indicators of healthy thriving dolphins. This group of dolphins is acoustically connected in open water lagoons in San Diego Bay. Communicative chatter of antiphonally exchanged whistles within the normal vocal range for the group may be considered a positive signal of the 'status quo'.

Acoustic monitoring for welfare does not have to be focused only on aquatic species. Automated monitoring systems are currently being developed and tested in densely populated enclosures of farm animals to improve welfare, decrease stress, and early identification of respiratory disease $[1,17,18]$. It is our hope that future projects can deploy similar systems for zoos and aquaria with terrestrial species. Early identification of atypical social interactions and/or distress calls in both aquatic and terrestrial species can lead to early intervention, better management of social groupings, and better overall health and welfare.

In conclusion, NMMF WAMS is freely available for use within PAMGuard opensource passive acoustic monitoring software. Please download NMMF WAMS from the PAMGuard plug-ins download page [19]. With its modular design, user-defined settings, and flexibility of use we look forward to seeing how researchers can creatively incorporate WAMS into their own works. It is our hope that zoos and aquaria will be inspired to innovate and create acoustic monitoring systems that fit the needs of their groups of animals and that the incorporation of acoustics becomes a common fixture for welfare monitoring.

Author Contributions: Conceptualization, B.L.J., M.O., M.B., S.T., J.M., S.H.R.; methodology, B.L.J., M.O., M.B., S.T., J.M., S.H.R.; software, M.O., J.M. validation, S.T., M.B.; investigation, B.L.J., S.T., M.B.; resources, S.H.R. data curation, M.B. writing-original draft preparation, B.L.J.; writing-review and editing, M.O., M.B., S.T., J.M., S.H.R.; supervision, S.H.R.; funding acquisition, S.H.R. All authors have read and agreed to the published version of the manuscript.

Funding: The authors are extremely grateful to the Office of Naval Research (ONR) for their support of Sound as Indicators of Health and Welfare of the Navy's Dolphin; ONR Grant\# N00014-18-12643. The funders had no role in study design, data collection and analysis, decision to publish, or preparation of the manuscript.

Institutional Review Board Statement: The MMP is AAALAC-accredited and follows the national standards of the United States Public Health Service Policy on the Humane Care and Use of Laboratory Animals and the Animal Welfare Act. The MMP's animal care and use program is routinely reviewed by an institutional animal care and use committee (IACUC) and the Navy Bureau of Medicine and Surgery (BUMED). BUMED concurred with the approval of MMP IACUC protocol \#130-2018 and assigned NRD\#1134 to the protocol for this project.

Data Availability Statement: The datasets generated during and/or analyzed during the current study are available from the corresponding author on reasonable request.

Acknowledgments: The authors are grateful for all of the hard work of the training staff for their continued dive support in deployment and retrievals of the underwater array. Thank you to the Navy Marine Mammal Program for their continued support for this project, and their dedication to the health and welfare of the Navy dolphins. We thank Doug Gillespie for his support and advisement throughout the project and Risa Daniels for her substantial contribution to the creation, maintenance and deployment of WAMS. This is the National Marine Mammal Foundation's contribution number 302 contribution to the scientific literature. 
Conflicts of Interest: The authors declare that they have no conflicts of interest to report. The funders had no role in the design of the study; in the collection, analyses, or interpretation of data; in the writing of the manuscript, or in the decision to publish the results. All of the authors were directly involved in the creation of the NMMF WAMS system.

\section{Appendix A}

Table A1. Whistle \& Moan Parameter Definitions as defined in Gillespie et al. [13]. For additional details please see Gillespie et al. [13].

\begin{tabular}{|c|c|}
\hline Setting & Description \\
\hline Maximum and Minimum Frequency & Defines the frequency range within which whistles will be detected. \\
\hline Connection Type & Which pixels will be examined when trying to trace out a whistle fragment. \\
\hline \multicolumn{2}{|c|}{$\begin{array}{l}\text { Connection Type } 4 \\
\text { Indicates that the algorithm will try to connect with the pixel above, below, to the left or to the right of the current pixel. } \\
\text { Connection Type } 8 \\
\text { Also adds the diagonal pixels to the potential search area. }\end{array}$} \\
\hline Minimum Length & $\begin{array}{l}\text { Whistle fragments shorter than the minimum length (measured in time slices, } \\
\text { or } \mathrm{ftt} / \mathrm{F}_{\mathrm{s}} \text { ) will be discarded. }\end{array}$ \\
\hline Minimum Total Size & $\begin{array}{l}\text { Whistle fragments shorter than the minimum total size (measured in number } \\
\text { of pixels) will be discarded. }\end{array}$ \\
\hline Crossing/Joining & How the Whistle \& Moan Detector deals with overlapping whistles. \\
\hline \multicolumn{2}{|r|}{ Leave branched regions intact } \\
\hline $\begin{array}{l}\text { Branched regions wi } \\
\text { Any region that has more th } \\
\text { All branches }\end{array}$ & $\begin{array}{l}\text { intact and may contain more than one actual sound. } \\
\text { Discard branched joins } \\
\text { detected frequency peak in any time slice will be discarded. } \\
\text { Separate all branches } \\
\text { separated and passed on as individual sounds. } \\
\text { Relink across joins }\end{array}$ \\
\hline \multicolumn{2}{|c|}{$\begin{array}{l}\text { The algorithm will attempt to re-join individual tones across joins. The sound is first broken up as above, and the individual } \\
\text { fragments are then re-joined according to a set of rules (see the help files for details). }\end{array}$} \\
\hline Max Cross Length & $\begin{array}{l}\text { Threshold used when analyzing crossed whistles. Where whistles cross, two } \\
\text { separate branches of a connected region merge, then re-separate. If the } \\
\text { number of time partitions in the merged section is less than the Max Crossing } \\
\text { Length, then it is assumed that this is indeed a genuine whistle crossing and } \\
\text { the connected region is separates into two whistles. If the merged region is } \\
\text { longer than this, then the region is split into four separate component } \\
\text { (the two entering the merged region and the two leaving it). }\end{array}$ \\
\hline Median Filter Length & $\begin{array}{l}\text { Within each spectrogram slice, the median value about each point is } \\
\text { calculated and subtracted from that point. The larger the median filter length, } \\
\text { the greater the number of points used to calculate the median. }\end{array}$ \\
\hline Average Subtraction Update Constant & $\begin{array}{l}\text { A decaying average spectrogram is computed and subtracted from the } \\
\text { current spectrogram value. The smaller the number, the greater/longer } \\
\text { the averaging. }\end{array}$ \\
\hline Threshold & A threshold value (in $\mathrm{dB}$ ) below which data is set to 0 . \\
\hline
\end{tabular}

\section{References}

1. Mcloughlin, M.P.; Stewart, R.; McElligott, A.G. Automated bioacoustics: Methods in ecology and conservation and their potential for animal welfare monitoring. J. R. Soc. Interface 2019, 16, 20190225. [CrossRef] [PubMed]

2. Brando, S.; Broom, D.M.; Acasuso-Rivero, C.; Clark, F. Optimal marine mammal welfare under human care: Current efforts and future directions. Behav. Process. 2018, 156, 16-36. [CrossRef] [PubMed]

3. Clegg, I.; Borger-Turner, J.L.; Eskelinen, H.C. C-Well: The development of a welfare assessment index for captive bottlenose dolphins (Tursiops truncatus). Anim. Welf. 2015, 24, 267-282. [CrossRef]

4. Jones, B.; Zapetis, M.; Samuelson, M.M.; Ridgway, S.H. Sounds produced by bottlenose dolphins (Tursiops): A review of the defining characteristics and acoustic criteria of the dolphin vocal repertoire. Bioacoustics 2019, 29, 399-440. [CrossRef] 
5. Moore, S.E.; Ridgway, S.H. Patterns of dolphin sound production and ovulation. Aquat. Mamm. 1996, 22, 175-184.

6. Ridgway, S.H. Dolphin hearing and sound production in health and illness. In Hearing and Other Senses; Far, R.R., Gourevitch, G., Eds.; Amphora Press: Groton, CT, USA, 1983; pp. 247-296.

7. Overstrom, N.A. Association between burst-pulse sounds and aggressive behavior in captive Atlantic bottlenosed dolphins (Tursiops truncatus). Zoo Biol. 1983, 2, 93-103. [CrossRef]

8. Caldwell, M.C.; Caldwell, D.K. Individualized whistle contours in bottlenosed dolphins (Tursiops truncatus). Nature 1965, 207, 434-435. [CrossRef]

9. Kuczaj, S.A., II; Frick, E.E.; Jones, B.L.; Lea, J.S.E.; Beecham, D.; Schnöller, F. Underwater observations of dolphin reactions to a distressed conspecific. Learn. Behav. 2015, 43, 289-300. [CrossRef] [PubMed]

10. Lilly, J.C. Distress call of the bottlenose dolphin: Stimuli and evoked behavioral responses. Science 1963, 139, 116-118. [CrossRef] [PubMed]

11. Blomqvist, C.; Amundin, M. High-frequency burst-pulse sounds in agonistic/aggressive interactions in bottlenose dolphins, Tursiops truncatus. In Echolocation in Bats and Dolphins; Thomas, J.A., Moss, C.F., Vater, M., Eds.; University of Chicago: Chicago, IL, USA, 2004; pp. 425-431.

12. Vannoni, E.; Torriani, M.V.G.; McElligott, A.G. Acoustic signaling in cervids. Cogn. Brain Behav. 2005, 9, 551-565.

13. Gillespie, D.; Caillat, M.; Gordon, J.; White, P. Automatic detection and classification of odontocete whistles. J. Acoust. Soc. Am. 2013, 134, 2427-2437. [CrossRef] [PubMed]

14. Rankin, S.; Archer, F.; Keating, J.L.; Oswald, J.N.; Oswald, M.; Curtis, A.; Barlow, J. Acoustic classification of dolphins in the California current using whistles, echolocation clicks, and burst pulses. Mar. Mamm. Sci. 2016, 33, 520-540. [CrossRef]

15. Houser, D.S.; Yost, W.; Burkard, R.; Finneran, J.J.; Reichmuth, C.; Mulsow, J. A review of the history, development and application of auditory weighting functions in humans and marine mammals. J. Acoust. Soc. Am. 2017, 141, 1371-1413. [CrossRef] [PubMed]

16. Southall, B.; Finneran, J.J.; Reichmuth, C.; Nachtigall, P.E.; Ketten, D.R.; Bowles, A.E.; Ellison, W.T.; Nowacek, D.P.; Tyack, P.L. Marine mammal noise exposure criteria. Aquat. Mamm. 2019, 45, 125-232. [CrossRef]

17. Schön, P.C.; Puppe, B.; Manteuffel, G. Automated recording of stress vocalisations as a tool to document impaired welfare in pigs. Anim. Welf. 2004, 13, 105-110.

18. Silva, M.; Ferrari, S.; Costa, A.; Aerts, J.M.; Guarino, M.; Berckmans, D. Cough localization for the detection of respiratory diseases in pig houses. Comput. Electron. Agric. 2008, 64, 286-292. [CrossRef]

19. Pamguard. Available online: https://www.pamguard.org/downloads.php?cat_id=9 (accessed on 1 June 2020). 